\title{
NEAR-INFRARED EMISSION-LINE SPECTRA OF THE ORION NEBULA, NGC 4151, AND OTHER SEYFERT GALAXIES
}

\author{
D. E. OSTERBROCK, R. A. SHAW, and S. VEILLEUX \\ Lick Observatory \\ University of California, Santa Cruz, CA 95064
}

\section{High Resolution Spectra}

CCDs make the near-infrared spectral region out to about $1.1 \mu$ observable. A good deal of information on the spectra of AGNs in this range has been published, which we are trying to systematize and extend at Lick. Our original lens-grism spectrograph camera is opaque over the region $\lambda 8600-\lambda 10250$, no doubt as a result of a coating on one of the elements of the Nikon lens, making this program impossible with it. However our newer "UV-Schmidt" camera is quite effective in this region. With it we have obtained well exposed spectra at moderate resolution $(F W H M \approx 7 \AA$ ) of NGC 4151 in three overlapping segments covering the region 7000-11000 $\AA$. Also, for comparison we obtained similar spectra of NGC 1976, the Orion Nebula. The wavelengths and relative line fluxes were measured, and most of the lines were identified. The strongest three lines in both objects are, in order, [S III] $\lambda 9532, \mathrm{He}$ I $\lambda 10830$, and [S III] $\lambda 9069$. The Orion Nebula spectrum is very helpful for identifying lines in NGC 4151, and for comparison with it. Among the lines identified in NGC 1976, many of them previously known, are [O II], [S II], [Ar III], [Fe II], [Ni II], [Ni III], [C $\ell$ II], [C I], in addition to O I $\lambda 8446$, many $\mathrm{H}$ I Paschen lines and $\mathrm{He} \mathrm{I}$.

Likewise in NGC 4151 [C I], [Fe II], [Fe XI], [C $\ell$ II], [Ni II], [Ar III], [Ar V], [S II] and [S VIII] are all present, in addition to O I $\lambda 8446, \mathrm{H} \mathrm{I}, \mathrm{He}$ I and He II. The coronal line [S VIII] $\lambda 9911$ has its ionization potentials between those of [Fe X] and [Fe XIV]. The strongest still unidentified emission lines in NGC 4151 are $\lambda \lambda 7045,9198$.

\section{Low Resolution Spectra}

We also obtained lower resolution spectra $(F W H M \approx 15 \AA$ ) of fifteen additional Seyfert galaxies, covering the range $\lambda \lambda 7000-10000$. [S III] $\lambda 9532$ is the strongest line in all of them but I Zw 1. Most of them show the well known strongest lines of [Ar III] and [O II]; nearly all of them show the strong [Ni II] line $\lambda 7378$; and many of them show O I $\lambda 8446$. Comparison of line strengths among these various Seyfert galaxies allows their ionization behavior to be traced. For this discussion we also used the large number of measurements of spectra on this region published by Morris and Ward (1988). Other still unidentified emission lines measured in the spectra of two or more Seyfert galaxies include $\lambda 7863$ (evidently a high-ionization line), $\lambda 9138$, and $\lambda 9189$ (evidently lower-ionization lines).

Some idea of the accuracy of observed relative line intensities may be obtained by 
comparison of theoretical and measured intensities of two lines from the same upper level. The calculated ratios depend only on ratios of transition probabilities, which are relatively well determined. For [S III] $\lambda 9532 / \lambda 9069$ the calculated ratio is 2.5 , our average measured value is $2.7 \pm 0.7$, and Morris and Ward found $2.4 \pm 0.7$. For [Ar III] $\lambda 7136 / \lambda 7751$ the calculated ratio is 4.1 , our measured average is $4.2 \pm 1.0$ and Morris and Ward found $2.8 \pm 1.1$. (In each case the standard deviation of a single measured ratio from the mean is given).

\section{Physical Conditions}

The main use of the near-infrared spectral measurements of AGNs is to extend the comparison with computed models over a greater number of ions and lines. This will require combining our measurements with measurements in the optical region. We are now in the course of measuring the optical spectra of all the objects for which we do not already have these data. The main problem is linking up the optical and near-infrared spectra.

One interesting comparison is the line ratio [Ar III] $\lambda 7136 /$ [S III] $\lambda 9069$, which is relatively independent of ionization level. For the AGN samples both we and Morris and Ward (1988) find the mean value $0.26 \pm 0.11$, while for six planetary nebulae with published spectra the same ratio is $0.55 \pm 0.32$. Thus, though the ranges overlap there is a significant difference in mean values. Whether this is an abundance effect, or an effect dependent on the shape of the photoionizing spectra will have to wait to be settled by computed models.

Also, for two of these objects for which we have prior measured data in the optical region, the $[\mathrm{O}$ III] $(\lambda 4959+\lambda 5007) / \lambda 4363$ and the analogous $[\mathrm{S} \mathrm{III]}(\lambda 9069+\lambda 9532) / \lambda 6312$ ratio can be solved simultaneously for the mean temperature and electron density in the $\mathrm{O}^{++}, \mathrm{S}^{++}$zone. The results are, for NGC $4151, T=13,000^{\circ}, N_{e}=3 \times 10^{5} \mathrm{~cm}^{-3}$; and for Mrk $3, T=9,200^{\circ}, N_{e}=6 \times 10^{5} \mathrm{~cm}^{-3}$. For the third object for which we have measurements, III $\mathrm{Zw} 77$, no common solution is possible; the [O III] ratio, which is unusually low, indicates a higher temperature than the [S III] ratio at all assumed densities. Because of the long wavelength interval between $\lambda 9532$ and $\lambda 6312$, and the fact that the latter line is often blended with the stronger [O I] $\lambda 6300$, [S III] is not as well suited as [O III] for such determinations.

\section{The Densest AGN}

I $\mathrm{Zw} 1$ is the only Seyfert galaxy we have observed in the near infrared in which there is no sign of [S III] $\lambda \lambda 9069,9532$ emission. This is in complete agreement with the fact that only very weak forbidden [O III] lines are seen in the optical region, and confirms that the electron density is unusually high in the "narrow-line region." From the absence of [S III] and the great weakness of [O III], the mean electron density in the NLR in I $\mathrm{Zw} 1$ probably is $N_{e} \approx 10^{8} \mathrm{~cm}^{-3}$.

Our spectra confirm the presence of [Ca II] $\lambda \lambda 7294,7324$, first identified in I Zw 1 by Phillips (1976). They are well resolved and definitely narrower than the other (permitted) emission lines. The critical density for these lines is $N_{c} \approx 10^{7} \mathrm{~cm}^{-3}$, and from their presence probably again $N_{e} \approx 10^{8} \mathrm{~cm}^{-3}$. We also confirm the presence of strong O I $\lambda 8446$ 
and Ca II $\lambda \lambda 8498,8542,8662$ emission first identified in I Zw 1 by Persson and McGregor (1985). O I $\lambda 8446$ is excited by resonance fluorescence in the "broad-line region," as outlined by Grandi (1980) and Morris and Ward (1985). The permitted Ca II lines' relative intensities show that they are optically very thick.

In addition we confirm the presence of Fe II multiplet (73), indicated by Phillips (1976) and identified (in part) by Persson and McGregor. It is the only multiplet of Fe II in the near infrared spectral region that is predicted to be excited by the resonance fluorescence process first postulated by Wampler and Oke (1967) and studied by Sargent (1968) and Phillips (1978). In addition we identify Fe II $\lambda \lambda 7376,7067$, the two strongest unclassified lines of the laboratory spectrum of this ion. From their presence in the spectrum of I $\mathrm{Zw} 1$, they probably are also excited by resonance fluorescence.

\section{References}

Grandi, S. A. 1980, Ap. J., 238, 10.

Morris, S. L., and Ward, M. J. 1985, M.N.R.A.S., 215, 57P.

. 1988, M.N.R.A.S., 230, 639 .

Persson, S. E., and McGregor, P.J. 1985, Ap. J., 290, 125.

Phillips, M. M. 1976, Ap. J., 208, 37.

$$
\text { . 1978, Ap. J., 226, 736/ }
$$

Sargent, W. L. W. 1968, Ap. J., 152, L31.

Wampler, E. J., and Oke, J. B. 1967, Ap. J., 148, 695. 